\title{
Coloanal Anastomosis: A Helpful Technique in Difficult Situations
}

\author{
Mohamed Ali Nada, MD; Islam Hossameldin ElAbbassy, MD \\ Department of General Surgery, Ain Shams University, Cairo, Egypt
}

Coloanal anastomosis (CAA) is used to preserve anal defecation after proctectomy. It is a savior technique when there are difficulties while performing low rectal anastomosis.

The aim of the study: was to highlight some of the indications, technique and the outcomes of CAA to raise the awareness of the young generations to use it when needed.

Patients and methods: This was a retrospective study that included 21 patients that had proctectomy and hand-sewn CAA with covering loop ileostomy. Full history, preoperative examination, operative details, and the follow-up notes were collected from our sheets. All patients were operated on by the same team of surgeons using the same technique. Closure of ileostomy was performed two to six months after the primary procedure.

Results: Out of the 21 patients, eight (38\%) had benign lesions, whereas 13 (61.9\%) had low rectal tumors. Pelvic abscess and complete dehiscence of the anastomosis, burst abdomen, and dehydrarion and renal impairment each occurred in one patient $(4.76 \%)$. Three patients $(14.3 \%)$ developed anastomotic stenosis and four (19\%) developed mild to moderate incontinence. Two patients developed radiological leak $(9.5 \%)$ that was observed via thin enema study after the $6^{\text {th }}$ postoperative week and disappeared after the $12^{\text {th }}$ week. All patients had a good quality of life after reversal of the stoma.

Conclusion: There is a time intra-operatively that a colorectal surgeon has to accept that stapled anastomosis is not feasible and should change to hand-sewn CAA as it could be the safest solution for many intra-operative anastomotic problems.

Key words: Coloanal anastomosis, hand-sewn, ileostomy, rectal cancer, stapled.

\begin{abstract}
Introduction
Coloanal anastomosis (CAA) that was introduced in the 1970s had become part of the procedures used to preserve anal defecation after proctectomy. ${ }^{1}$ It was proposed for patients with low rectal cancers allowing sphincter preservation with normal anal continence when compared to abdominoperineal resection. ${ }^{2-4}$
\end{abstract}

CAA is a savior technique when there are difficulties while performing low rectal anastomosis; and unfortunately nowadays most of the young surgeons miss how to do it.

Thus, the aim of this study was to highlight some of the indications, technique and the outcomes of CAA to raise the awareness of the young generations to use it when needed.

\section{Patients and methods}

This is a retrospective study that was approved by the ethical and scientific committee, General Surgery Department, Ain-Shams University. The study included 21 patients that had proctectomy and hand-sewn CAA with covering loop ileostomy, during the period from 2011 until the end of 2015, at Ain Shams University Hospital, Cairo, Egypt. Full data including history, preoperative examination, operative details, and the follow-up notes were collected from our sheets. All patients were operated on by the same team of surgeons using the same technique. Closure of ileostomy was performed two to six months after the primary procedure.

\section{The operative technique:}

All patients were placed in Lloyd-Davis lithotomy position. The procedure started by proper mobilization of the colon (through the laparotomy incision) by ligating the inferior mesenteric artery at its origin and ligating the inferior mesenteric vein at the inferior border of the pancreas. This mobilization was done to facilitate making a tension-free CAA and was done with great care not to injure the marginal blood supply, as it was the only blood supply to the proximal anastomotic side. (Figure 1). 


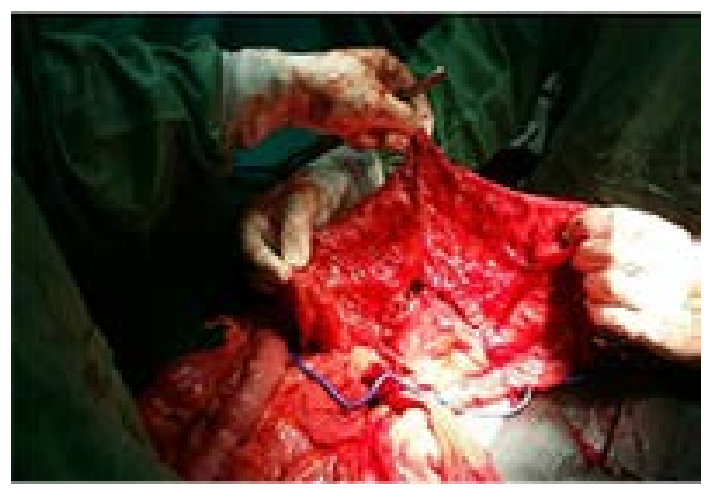

Fig 1: Adequate mobilization of the colon with preserving the marginal blood supply for tension-free hand-sewn CAA.

Dissection distal to the peritoneal reflection was continued to the level of the pelvic floor. The muscular rectal wall was divided at the level of the ano-rectal ring then the specimen was retrieved through the laparotomy incision, making sure that there was a proper blood supply at the proximal end, and then, four stay sutures (full thickness) at the proximal colonic edge were taken.

The perineal part of the procedure started by everting the anal canal using eight vicyl stitches $(1 / 0)$, instead of using the Loan Star retractor. This was followed by rectal mucosectomy (transanal dissection) in which the rectal mucosa was stripped from the dentate line. Mucosectomy was continued proximally till the distal cut end of the rectum. (Figures 2-4).

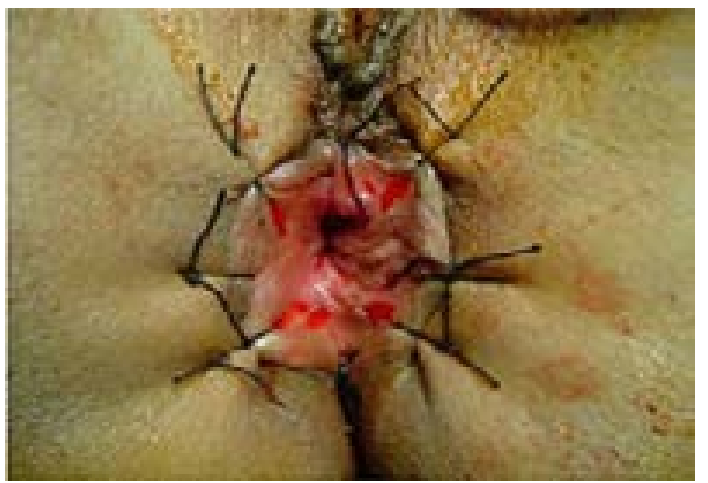

Fig 2: Everted canal using vicryl stitches.

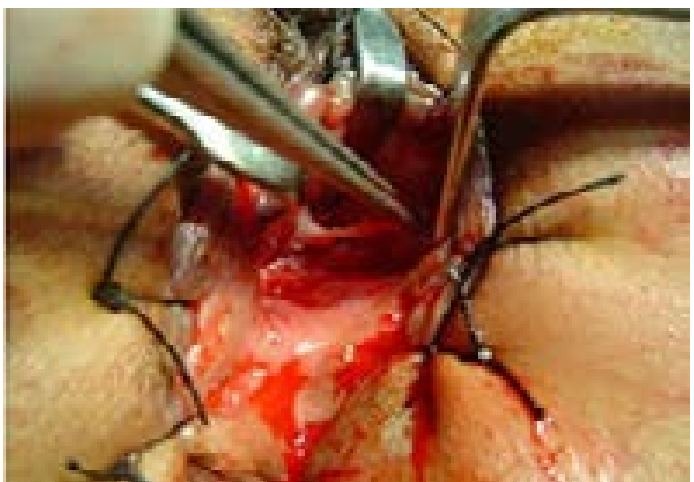

Fig 3: Performing rectal mucosectomy.

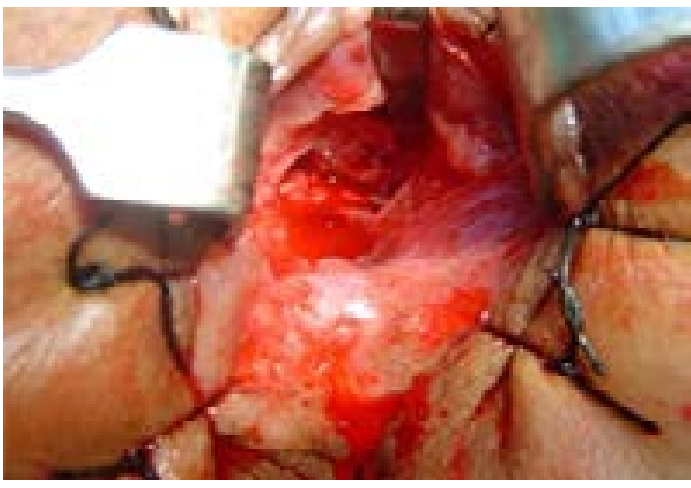

Fig 4: After copmpleting full rectal mucosectomy.

Stay sutures were taken in the distal stump (at 3, 6,9 and 12 o'clock) at the level of the dentate line and were pulled through the anus until the stump was partially everted.

The stay sutures in the proximal stump were pulled through the distal one until coming out from the anus, keeping its mesentery in its anatomical posterior position without twisting the colon. Any tension on the proximal colon during this step was avoided, allowing its passage through the anal canal smoothly to reach an adequate distance permitting an easy accessible anastomosis. (Figure 5).

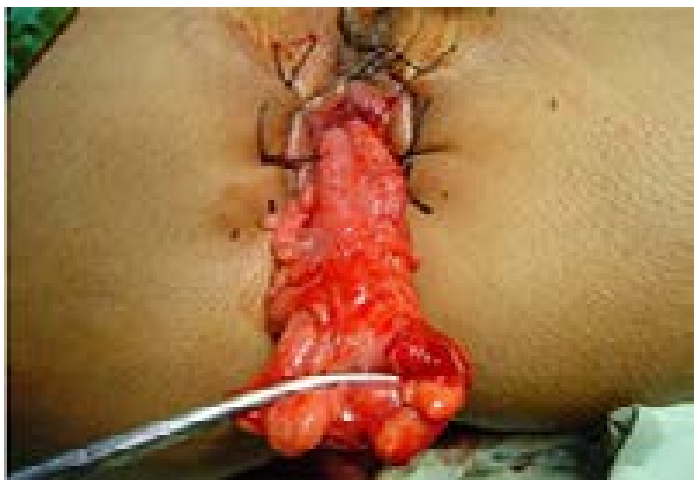

Fig 5: Passing the proximal colon out through the anus preserving its mesentery posteriorly.

Then straight end-to-end CAA was performed via trans-anal hand-sewn method (as described by Parks), ${ }^{1}$ using one layer of interrupted absorbable sutures beginning at the sites of the already fixed stay sutures, followed by quadrant by quadrant stitches. Stitches were taken in a special manner (mucosa at the dentate line with the underneath internal sphincter at one side, to full-thickness colonic wall at the other side). (Figure 6). 


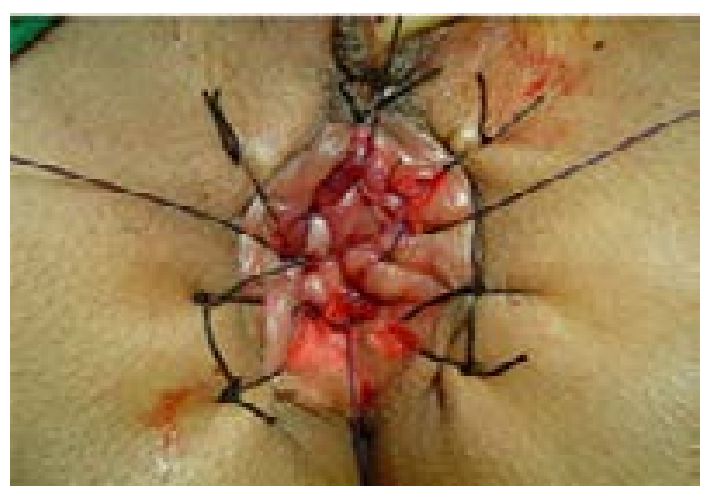

Fig 6: Performing CAA with mild tension over the four stay sutures.

After completing the anastomosis, the rectum was placed back into the pelvis followed by suturing the peritoneum of the mesentery to the pelvic wall to seal the pelvis off from the peritoneal cavity. (Figure 7) Then covering loop ileostomy was done.

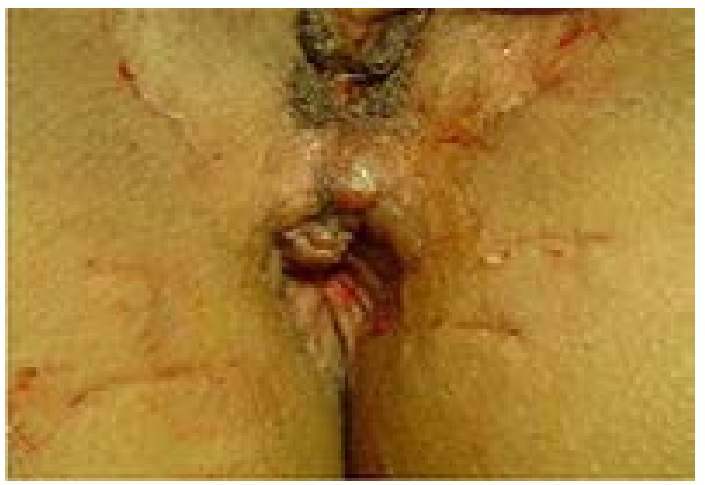

Fig 7: After completing the CAA.

\section{Results}

Twenty one patients were included in the study, nine of them were males (42.8\%) while 12 were females $(57.2 \%)$. Out of those 21 patients, eight $(38 \%)$ had benign lesions (two had megarectum, one had rectocutaneous fistula, one had benign stricture, one had retroprostatic fistula, two had multiple hamartomas and one had rectal adenoma); whereas 13 patients $(61.9 \%)$ had low rectal tumors. 11 out of them received preoperative radiochemotherapy (RCT).

The median age for the benign group was 29 years and for the malignant one was 37 years.

Pelvic abscess and complete dehiscence of the anastomosis happened on the fifth postoperative day in one patient $(4.76 \%)$ that had a rectal hamartomatous lesion. This was managed by end colostomy, then was operated on by pull-through procedure with total hospital stay of 17 days.
Burst abdomen occurred on the ninth postoperative day in one patient $(4.76 \%)$ with preoperative RCT that was managed by surgical intervention and wound closure with total hospital stay of five weeks.

Dehydration and renal impairment were diagnosed about ten days after discharge in one patient $(4.76 \%)$ with RCT who was readmitted for eight days and improved by medical treatment.

Three patients (14.3\%) developed anastomotic stenosis and anal dilatation was done in the outpatient clinic.

Four patients (19\%) developed mild to moderate degree of incontinence (Wexner score 4-6).

Two patients developed radiological leak (9.5\%) that was observed via thin enema study (delayed post-evacuation films) after the 6th postoperative week. However, after the 12th week, no leak was detected when the enema study was repeated.

At the end of the study, all patients were reported to have a good quality of life after reversal of the stoma.

\section{Discussion}

Coloanal anastomosis (CAA) was first introduced in the 1970 s by Sir Alan Parks. ${ }^{1}$ In 1982, Parks and Perey used this technique in patients with rectal cancer. ${ }^{5}$ In cases having low rectal cancer, a distal resection margin of two $\mathrm{cm}$ has been widely accepted and ultralow sphincter-saving rectal resection with CAA has been considered a good surgical choice, even in the lower one third of the rectum. ${ }^{6}$

In our study, the reason behind performing CAA for patients with benign diseases varied from one case to another.

Two patients $(9.5 \%)$ with megarectum and Hirshsprung disease had CAA because of having an aganglionic segment situated just above the dentate line. Therefore, to achieve a successful surgical technique, we had to do our anastomosis very deep and below the level that is accessible by the available staplers.

However, thickened distal stump by fibrosis with failed stapled stricturectomy was the reason for performing CAA in one patient (4.8\%) with a benign stricture.

Severe thickening of the distal rectum posteriorly in a patient with rectocutaneous fistula required a CAA. This was also required for another patient with a very huge rectocutaneous fistula just above 
the anorectal junction.

In a patient with rectal adenoma and two patients with rectal hamartomas, the staplers could not reach safely distal to the lesion, so CAA was done.

Moreover, there were different reasons for performing CAA in the 13 malignant cases included in the study.

One patient with advanced rectal cancer had CAA because the mass was huge and could not be resected from the abdomen, so combined abdominoperineal resection anastomosis was done according to the technique mentioned by Abou Zeid and Makki. ${ }^{7}$

In six patients with low rectal cancer and neoadjuvant RCT, CAA was done because of having very low tumors and we were not satisfied with the adequate length of the distal margin that would be reached if we used staplers.

In another three patients, we already performed stapling and resection of the distal end but we realized that we had an inadequate distal resection margin, so we completed more distal dissection until an accepted distal margin was reached, then we shaved the specimen from the top of the pelvic floor performing hand-sewn CAA.

CAA was done for a patient with malignant ulcer and neoadjuvant RCT as the distal stump was very thick due to radiation effect. CAA was also done for another patient after failure of stapling and leaking from the site of the staple line, so we disrupted the staple line and proceeded to CAA. However, the last malignant case had a very narrow pelvis that we could not introduce the stapler in, thus CAA was performed.

Sagar PM and his colleagues reported that performing CAA either hand-sewn or by using staplers did not affect the rate of recurrence in malignant cases. ${ }^{8}$

Regarding the complications, we detected one case $(4.76 \%)$ of anastomotic dehiscence, in spite of having a covering ileostomy that was presented with pelvic abscess. This percentage is quite lower than that described by Cavaliere et $\mathrm{al}^{9}$ and Cutait et $\mathrm{al}^{10}$ who reported anastomotic leak rate of $18 \%$ and $31.9 \%$ respectively, even with using a covering stoma. However, in a study done by Mohamed and his collegues in 2011, they detected leakage in three cases (4.2\%) after performing external CAA without covering stoma. Those cases were also presented locally with pelvic abscess without developing peritonitis. ${ }^{11}$ At the end of their study, they concluded that using a covering stoma with
CAA does not decrease the rate of anastomotic leakage. However, we believe that if a covering stoma does not prevent complications of leakage, it minimizes their severity, moreover, radiological leaks that would be discovered prior to closure of ileostomy, could be managed conservatively and most of them will close spontaneously if stoma closure was delayed for few weeks.

Three patients (14.3\%) in our study experienced stenosis of the CAA. This percentage is considered to be within the range reported by other studies that varied between $6.3 \%$ and $42.1 \% .^{9,12,13}$ Those patients were managed only by dilatation in our out-patient clinic without any need for more aggressive management. By comparing hand-sewn anastomosis to the stapled one, anal stenosis was reported to be higher in stapled anastomosis. ${ }^{14,15}$

However, in a study done by Cong and his colleagues on 22 patients in 2014, stapled CAA was found to cause anastomotic leakage and stenosis less significantly than the hand-sewn anastomosis. ${ }^{16}$

We also reported four patients (19\%) that developed mild to moderate degree of incontinence, however, frequent defecation was reported in around 30\% of patients included in other studies. ${ }^{11,17,18}$ This was explained by reducing the capacity of the rectal reservoir which was then increased with time due to progressive distention of the distal colon.

In many studies, anorectal continence after handsewn CAA was reported to be of low grade (Grade I or II)..$^{5,19-24}$ Also, Cong et al documented that there was no significant difference in Wexner score between stapled and hand-sewn CAA $(P>0.05) .{ }^{16}$

Moreover, it was documented in a study done by Horgan et al that hand-sewn anatomosis had a better impact on discrimination and recovery of the anal inhibitory reflex than the stapled one..$^{25}$ This was explained by the allowance of the handsewn stitches to let the nerves grow through the anastomosis, which is usually affected by the stapled anastomosis.

In a study done by Eichhoff $\mathrm{G}$, eight patients (14\%) developed colonic necrosis due to excessive mobilization of the colon. ${ }^{26}$

This problem was not reported in our study because we were keen on preserving the marginal blood supply and on making a tension-free CAA.

We had to highlight that hand-sewn CAA was not offered as an alternative to the traditional stapled anastomosis for low rectal diseases, but it was the only way of anastomosis in many 
unpleasant situations. Preoperative planning for it was missed and thus, it was an intra-operative decision. Unfortunately, the alternative is usually performing a permanent stoma rather than making an anastomosis.

\section{Conclusion}

Proctectomy with hand-sewn CAA is a safe, technically feasible, having an accepted rate of complications and has a good functional outcome.

There is a time intra-operatively that a colorectal surgeon has to accept that stapled anastomosis is not feasible and should change to hand-sewn CAA, especially when there is extensive fibrosis, extensive radiation effects, failure of stapling, narrow pelvis, inadequate distal safety margin, or if there are other substitutes that may end up in an unsafe anastomosis or having a permanent stoma.

Thus, we believe that it is mandatory for senior coloproctology surgeons to train the younger fellows on the technique of hand-sewn CAA even before gaining the experience of the stapled anastomosis, as it could be the safest and savior solution for many intra-operative anastomotic problems.

\section{References}

1. Nicholls RJ: Rectal cancer: Anterior resection with per anal colo-anal anastomosis. The results in 76 patients treated by Sir Alan Parks. Bull Cancer 1983; 70: 304-307.

2. Bittorf B, Stadelmaier U, Göhl J, Hohenberger W, Matzel KE: Functional outcome after intersphincteric resection of the rectum with coloanal anastomosis in low rectal cancer. EJSO 2004; 30 :260-265.

3. McNamara DA, Parc R: Methods and results of sphincter-preserving surgery for rectal cancer. Cancer Control 2003; 10: 212-218.

4. Bordeianou L, Maguire LH, Alavi K, Sudan $R$, Wise $P E$, Kaiser AM: Sphincter-sparing surgery in patients with low-lying rectal cancer: Techniques, oncologic outcomes, and functional results. J Gastrointest Surg 2014; 18: $1358-1372$.

5. Parks AG, Perey JP: Resection and sutured coloanal anastomosis for rectal carcinoma. $\mathrm{Br}$ J Surg 1982; 69 :301-304.

6. Chen JC, Chen JB, Wang HM: Laparoscopic Coloanal Anastomosis for low rectal cancer. JSLS 2002; 6: 345-347.

7. Abou-Zeid AA, Makki MT: Combined abdominal and perineal approach for delayed restoration of bowel continuity after low anterior resection in females. Dis Colon Rectum 2007; 50: 544547.

8. Sagar PM, Pemberton JH: Surgical management of locally recurrent rectal cancer. Br J Surg 1996; 83: 293-304.

9. Cavaliere F, Pemberton JH, Cosimelli M, Fazio VW, Beart Jr RW: Coloanal anastomosis for rectal cancer: long-term results at the Mayo and Cleveland Clinics. Dis Colon Rectum 1995; 38: 807-812.

10. Cutait $D E$, Cutait $R$, Ioshimoto $M$, Hyppólito da Silva J, Manzione A: Abdominoperineal endoanal pull-through resection. A comparative study between immediate and delayed colorectal anastomosis. Dis Colon Rectum 1985; 28: 294-299.

11. Mohamed AA, Abdel-Fatah AF, Mahran KM, Mohie-Eldin AB: External coloanal anastomosis without covering stoma in low-lying rectal cancer. Indian J Surg 2011; 73: 96-100.

12. Luna-Pérez $\quad P, \quad$ Rodriguez-Ramirez $S$, Hernandez-Pacheco $F$, Gutierrez De La Barrera M, Fernandez R, Labastida S: Anal sphincter preservation in locally advanced low rectal adenocarcinoma after preoperative chemoradiation therapy and coloanal anastomosis. J Surg Oncol 2003; 82: 3-9.

13. Bernard D, Morgan S, Tasse D, Wassef R: Preliminary result of coloanal anastomosis. Dis Colon Rectum 1989; 32: 580-584.

14. Keighley MRB, Williams NS: Surgery of the Anus, Rectum and colon. London, Philadelphia, Totonto, Sydney, Tokyo: W. B. Saunders Ltd. 1993.

15. Soreide O, Norstein J: Rectal cancer surgery. Berlin, Heidelberg, New York: Springer-Verlag 1997.

16. CongJC,ChenCS,MaMX,XiaZX,Liu DS,ZhangFY: Laparoscopic intersphincteric resection for low rectal cancer: Comparison of stapled and manual coloanal anastomosis. Colorectal Dis 2014; 16: 353-358.

17. Otto IC, Ito K, Ye C, Hibi K, Kasai Y, Akiyama $S$, et al: Causes of rectal incontinence after sphincter-preserving operations for rectal cancer. Dis Colon Rectum 1996; 39: 14231427. 
18. Williamson ME, Lewis WG, Finan PJ, Miller AS, Holdsworth PJ, Johnston D: Recovery of physiologic and clinical function after low anterior resection of the rectum for carcinoma: Myth or reality? Dis Colon Rectum 1995; 38: 411-418.

19. Schiessel R, Karner-Hanusch J, Herbst F, Teleky $B$, Wunderlich $M$ : Intersphincteric resection for low rectal tumours. Br J Surg 1994; 81: 13761378.

20. Rullier E, Sa CA, Couderc P, Rullier A, Gontier R, Saric J: Laparoscopic intersphincteric resection with coloplasty and coloanal anastomosis for mid and low rectal cancer. Br J Surg 2003; 90: 445-451.

21. Baik SH, Kim NK, Lee KY, Sohn SK, Cho CH: Handsewn coloanal anastomosis for distal rectal cancer: Long-term clinical outcomes. J Gastrointest Surg 2005; 9: 775-780.
22. Keighley MR, Matheson D: Functional results of rectal excision and endo-anal anastomosis. Br J Surg 1980; 67: 757-761.

23. Rouanet $P$, Fabre JM, Dubois JB, Dravet $F$, Saint $A B$, Pradel J, et al: Conservative surgery for low rectal carcinoma after high-dose radiation. Functional and oncologic results. Ann Surg 1995; 221: 67-73.

24. Schiessel R, Wunderlich M, Waneck R: Results of coloanal anastomosis in deep-seated tumors of the rectum. Chirurg 1986; 57: 792-796.

25. Horgan PG, O'Connell PR, Shinkwin CA, Kirwan WO: Effect of anterior resection on anal sphincter function. Br J Surg 1989; 76: 783786.

26. Eichhoff G: Short and long-term results of hand-sewn coloanal anastomosis performed as a salvage procedure after rectal resection. IJS 2008; 18: 1. 